\title{
A New Model Describing the Coordination Pattern of the Legs of a Walking Stick Insect *
}

\author{
H. Cruse \\ Fachbereich Biologie der Universität Kaiserslautern, FRG
}

\begin{abstract}
A computer (Fortran) model is proposed that describes the temporal and spatial coordination pattern of straight walking stick insects (Carausius morosus) for a broad speed range. It provides a stable pattern independent of the different starting positions. The model is based on six relaxation oscillators. The leading oscillator corresponds to a frontleg. Therefore the information flow runs from front to rear in contrast to earlier models (Graham, 1972; Wendler, 1968).
\end{abstract}

\section{Introduction}

If one is interested in the question "how are the movements of the legs in walking insects coordinated?" one possible method of investigation is to construct a model, that describes the coordination of the legs. To construct such a model one has to introduce hypotheses on the kind of the six subsystems and of connections between the six subsystems, each of which controls the movement of one leg. By the comparison of the model results with the experimental results these hypotheses can be tested. Additionally this comparison may suggest new experiments providing a test of the hypothesis. In the literature two quantitative models exist (Graham, 1972, 1977a; Wendler, 1968, 1978) and some qualitative models (e.g. Hughes, 1957; Wilson, 1966). The qualitative models are based on experimental results of cockroaches, the models of Wendler and Graham are based on the behaviour of the stick insect Carausius morosus. The last mentioned has been extended to include the behaviour of decerebrated and amputated stick insects (Graham, 1977b, 1979) and of other Orthoptera and Blattodea (Graham, 1977a, cockroach, locust ; Graham, 1978a, b, grasshopper Neoconocephalus robustus).

* Supported by DFG
According to Wilson (1966) the coordination pattern of an insect is represented in the following way (Fig. 1). The abscissa shows the time, the six traces show the six legs in the order R3 (right hindleg), R2 (right middleleg), R1 (right frontleg), L3 (left hindleg). L 2 (left middleleg) and L1 (left frontleg) from top to bottom. The black bars show the duration of the protraction phase (swing phase). The white parts between represent the retraction phase (stance phase). All the authors cited above begin with the observation, as can be seen in Fig. 1, that on each side of the animal there is a metachronal wave of protraction, which runs from back to front (surrounded by solid lines). This observation brings the authors to the assumption, that there is a directly corresponding physiological relation in the sense that a protraction in the hindlegs is followed after a definite time delay by a protraction in the ipsilateral middleleg and after some appropriate time delay this is followed by a protraction of the ipsilateral frontleg. The information flow for this hypothesis runs anteriorly. An other support for this assumption probably was the evolutionary argument that also in many Annelids and Chilopods such metachronal waves travelling towards the front end of the body can be seen.

In Fig. 1, however, there can also be seen a metachronal wave in the opposite direction (surrounded by interrupted lines). Because of the laws of Gestalt psychology it is not so immediately obvious. In the following it will be shown, that models using a posteriorly directed information flow can be con-

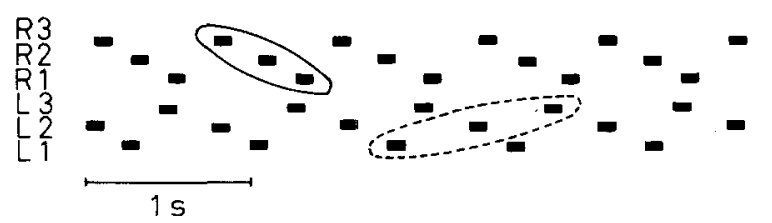

Fig. 1. Typical coordination pattern of an adult stick insect (from Graham, 1972) 


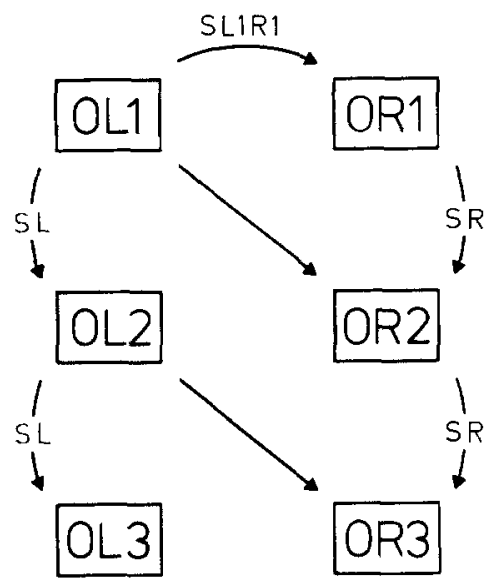

Fig. 2. A schematic drawing showing the hypothetical connections between the six subsystems, each of which controls the movement of one leg. The symbols for the different switches correspond to those shown in Fig. 3. For further explanations see text

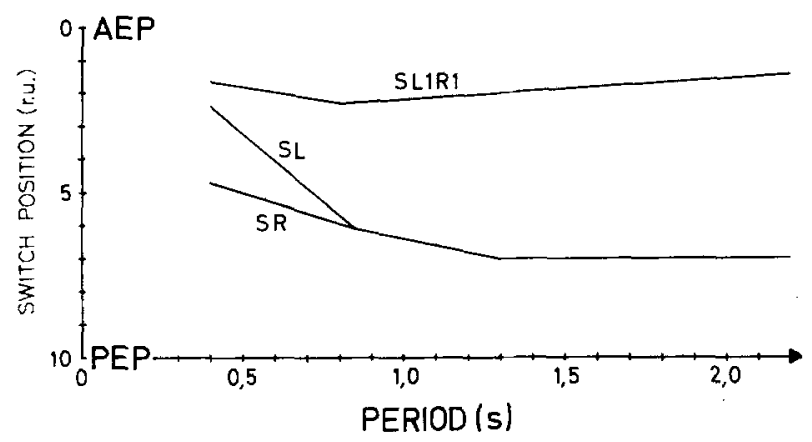

Fig. 3. The position of the switch in the controlling leg as a function of stepping period. The switch position is given as a decimal fraction of the ten unit interval between anterior extreme position (AEP) and posterior extreme position (PEP). SL: switch between OL1-OL2 and between OL2-OL3. SR: switch between OR1-OR2 and between OR 2-OR3. SL1R1: switch between OL1-OR1. For further explanations see the text

structed. Because the most detailed data are available from stick insects, the model will be based mainly on experimental results from these animals.

\section{The Model}

In order to construct the model two different mechanisms are used. It is shown experimentally that the mechanism described first is generally existent in insects: A single leg can show a normal stepping behaviour when phasic influence from the other legs is either absent or incorrect. This is shown in the following situation. If a stick insect walks on a treadmill and all the legs except one are resting on sticks fixed relative to the insects body, each leg tries to press its own stick in a rearward direction but because of the rigidity of the stick only very small movements are possible. Nevertheless the only leg remaining on the treadmill performs qualitatively normal steps (Cruse and Schwarz, 1979). Therefore the following hypothesis is assumed. Each leg has a geometrically defined posterior extreme position (PEP). If the leg reaches its PEP (probably determined by sense organs), the leg normally (for exceptions see below) starts a protraction. This hypothesis is supported by observations of Bethe (1897), Ten Cate (1928) and Matula (1911) [all cited after Wilson (1966), p. 112] on cockroaches and of Bässler $(1967,1977)$ on stick insects. A particular good example of this is the observation of Ten Cate (1936), that if an isolated thoracic segment of a cockroach is dragged along a surface, the leg steps each time it is stretched a certain distance relative to the body. Correspondingly each leg is assumed to have an anterior extreme position (AEP). This is supported by results of Wong and Pearson (1976), who showed that the stimulation of the trochanteral hair sensilla inhibits further flexion of the coxa-trochanterofemurjoint (which corresponds to protraction in cockroaches) and removal of these sensilla provides a forward displacement of the AEP of this leg. Therefore in the model it is assumed, that every leg works like a relaxation oscillator, i.e. if the leg reaches its PEP, it protracts until it reaches its AEP, than swings back again. Although these results might support the assumption, that the extreme positions (AEP and PEP) are determined by sensory feedback, up to now it is not clear whether they might also be controlled by centrally produced time delays (see also discussion).

There are probably different types of influences between any two subsystems, each of which controls the movement of one leg. Wilson (1966) observed that the PEP of a grasshoppers hindleg is not fixed relative to the body, but the time of protraction is triggered by an influence of the other legs. This observation has been quantitatively examined by Graham $(1978 \mathrm{a}, \mathrm{b})$ and supports the above hypothesis. How this influence operates is assumed in a second hypothesis: there exists a switch, that is turned on when the "leg" has a defined position and a defined direction of movement. The switch is used to initiate a protraction in another "leg" by lowering the threshold of the relaxation oscillator mentioned above. If the latter "leg" therefore has reached a position sufficiently far behind, a protraction begins.

From these two assumptions a quantitative model written in Fortran (using a time raster of $20 \mathrm{~ms}$ ) is constructed in the following way. Each of the six legs is represented by a relaxation oscillator, which is named by putting an $\mathrm{O}$ in front of the abbreviation of the corresponding leg (e.g. OL1, OR1). Describing the retraction movement, the oscillator increases its output value at a defined rate, that corresponds to the walking speed of the animal. When its output reaches a 
threshold (corresponding to the PEP of the leg), it returns to zero (corresponding to the AEP of the leg) by a constant rate. This rate is chosen to be constant as in the normal gait of an adult stick insect [named gait II by Graham (1972)] the protraction time is always about $100 \mathrm{~ms}$ independent of the walking speed. The output value of the relaxation oscillator describes the position of the tarsus of the leg projected on the longitudinal axis of the body. While the zero value corresponds to the AEP, the normal threshold to start the protraction is chosen to be $T_{0}$. If no influences of the other legs occur to change this threshold, the value $T_{0}$ describes the position of the PEP of the corresponding leg.

Different pairs of oscillators are connected in such a way that when in the retraction phase the output value of the first oscillator increases and reaches a definite value ("switch position"), the threshold of the second oscillator is decreased to a defined value $T_{1}$. The first oscillator is called the "controlling oscillator", the second one is called the "controlled oscillator". In the model such connections are assumed to exist from OL1 to OL2, from OL2 to OL3, from OL1 to OR 1, from OR 1 to OR2 and from OR2 to OR3. In Fig. 2 these connections are symbolized by curved arrows. From the data of Graham (1972), the exact position of these switches must depend on the walking speed. As the speed increases the "switch position" moves anteriorly. In Fig. 3 this dependence is shown for the different switches used in the model. The stepping period (time of protraction plus time of retraction) is given by the abscissa and the switch position of the controlling oscillator is shown on the ordinate. These functions are partly derived directly from data of Graham (1972, Fig. 8), partly they are obtained from the model calculation when adapting the model data to the experimental results of Graham (1972).

Some authors (e.g. Wilson, 1966; Graham, 1972) mention, that pair of legs L1-R 2 are in phase at all walking speeds. That means the protraction of both legs occurs at the same time. The same is stated for the pair L2-R3. Therefore in the model an across the body connection between OL 1 and OR 2 and between OL 2 and OR 3 is assumed, and during the protraction of OL1 the threshold of OR 2 is lowered to the value $T_{2}$. The same connection is constructed between OL2 and OR 3. Both connections are symbolizéd in Fig. 2 by straight arrows. As the results show, with these two additional connections the connections between OR 1 and OR 2 and between OR 2 and OR 3 are no more necessary. Therefore one might argue that they can be deleted. However Graham $(1972,1979)$ could show that the three legs on either side of the body can walk in normal ipsilateral coordination without any coupling between right and left sides. Therefore one must assume that the three cross-connections in the model can be switched off by a central mechanism. In this case the connections between OR 1 and OR 2 and between OR 2 and OR 3 are still necessary to produce a proper coordination between the legs of the right side of the body.

\section{Results}

When testing such a model, attention must first be concentrated upon the question, whether or not the model creates a stable coordination pattern when a) different combinations of initial values of the six oscillators occur (which corresponds to different starting positions of the legs) and when b) the walking speed is varied. To obtain stable patterns it was a necessary condition that the values of the eigenfrequencies of the six oscillators are arranged in a definite order which is explained in detail below. When the values of the different parameters were adjusted in a certain manner, the model showed a stable pattern for each walking speed independent of the particular starting position. Additionally it describes the pattern of the walk of an adult stick insect as it will be shown in the following.

To describe the dependence of the coordination pattern on the walking speed, Graham (1972) measured the time lag between the beginning of protraction of leg $\mathrm{L} 3$ and the beginning of the following protraction of leg $\mathrm{L} 1$. The same was done for the corresponding legs on the right side of the body. These time lags were plotted against period. The period is the time between the beginning of protraction of one leg and the beginning of the next protraction of the same leg, and is the same for all six legs when a stable coordination pattern occurs.

Adult stick insects can use two different types of gait, which are called gait I and gait II by Graham (1972). In Fig. 4 the lag values measured by Graham for gait II are compared with the corresponding values of the model. As can be seen, the values of the proposed model lie within the values of the standard error of the mean of Graham's measurements. The real behaviour of the model can be seen in Fig. 5, where three examples of "walks" at different speeds are shown.

To show the stability of the model, the possible ranges of the parameters must be given. Within these ranges the coordination pattern is independent of the exact value of the individual parameters. The eigenfrequency of such an oscillator can be changed either by changing the slope of the output or by changing the threshold. In this model changing the slope results in a slightly different retraction speed and because of the constant time relations results in a change of the posterior extreme position of the different legs. These effects do not occur when the thresholds are changed. In the model this second method is used. (The first method 


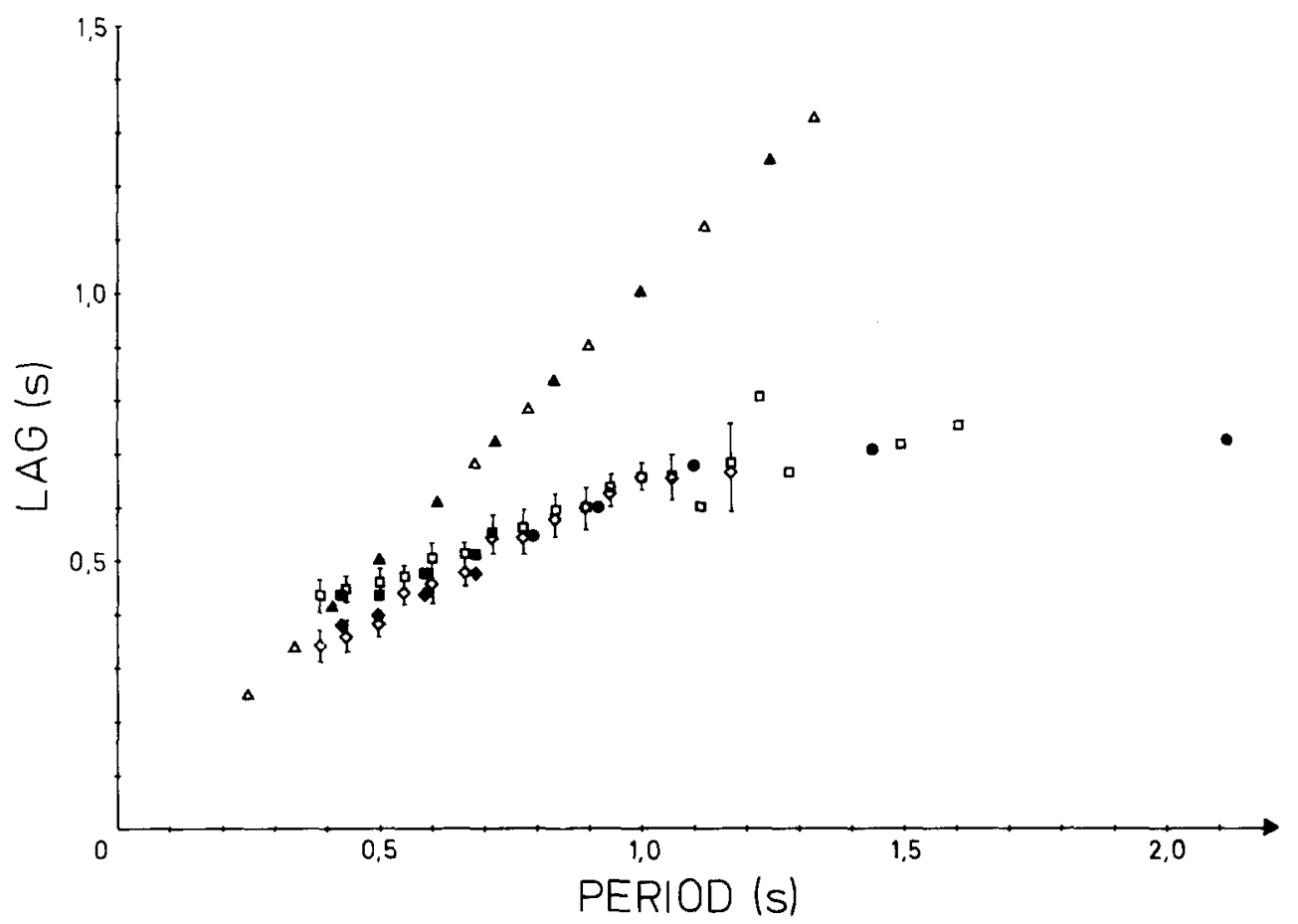

Fig. 4. Comparison of experimental results of Graham (1972) for gait I ( $\triangle$ ) and gait II ( $\diamond$ lag of the right legs, $\square$ lag of the left legs) with the data obtained by the model. The lag is measured as the time interval between the beginning of protraction in the hindleg and the immediately following protraction in the ipsilateral front leg. For gait II the vertical bars show the error of the mean of the experimental results. Individual measurements show no bars. For gait I the error of the mean is smaller than the symbols. The model values are given by the corresponding closed symbols. If there is no difference between lag of the right and the left side for gait II the values are shown by circles

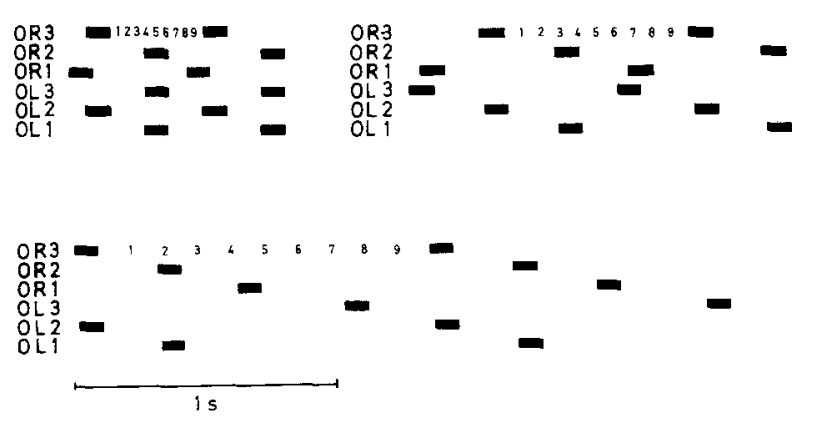

Fig. 5. The behaviour of the model when describing the coordination pattern of gait II for three different walking speeds. As in Fig. 1 the black bars show the protraction phases of the legs. The numbers on the retraction phase of OR 3 show the output values of the oscillator, describing the position of the equivalent tarsus

was also investigated and seemed to show no essential differences.) The threshold which determines the eigenfrequency of the oscillator is called $T_{0}$. To obtain stable patterns, the value $T_{0}$ of the oscillator OL 1 must be the lowest one of all and is fixed in all calculations at the value of 10 . The values in Table 1 show the lowest thresholds $T_{0} \min$ for the other oscillators capable of producing a stable coordination pattern. This corresponds the observation of Wendler (1964) that the frequency of the frontleg is higher than that of the hindleg when the insect is supported upon a treadmill
Table 1. The parameter ranges used in the model which give a constant coordination pattern independent of the different starting positions

\begin{tabular}{lcccccc}
\hline & OL 1 & OL2 & OL 3 & OR 1 & OR2 & OR 3 \\
\hline$T_{0} \min$ & 10 & 10.2 & 10.2 & 10.2 & 10.3 & 10.3 \\
\hline
\end{tabular}

$T_{1}:$ 5.-9. $\quad T_{2}: 1 .-10$.

Table 2. The different starting positions of the oscillators used in the model calculation

\begin{tabular}{llllllllllr}
\hline Position no. & 1 & 2 & 3 & 4 & 5 & 6 & 7 & 8 & 9 & 10 \\
\hline OR 3 & 1 & 2 & 4 & 6 & 8 & 9 & 9 & 2 & 7 & 1 \\
OR 2 & 1 & 2 & 4 & 6 & 8 & 9 & 4 & 5 & 5 & 10 \\
OR 1 & 1 & 2 & 4 & 6 & 8 & 9 & 2 & 8 & 3 & 2 \\
OL 3 & 1 & 2 & 4 & 6 & 8 & 9 & 8 & 8 & 7 & 1 \\
OL 2 & 1 & 2 & 4 & 6 & 8 & 9 & 5 & 5 & 5 & 5 \\
OL 1 & 1 & 2 & 4 & 6 & 8 & 9 & 1 & 1 & 3 & 7 \\
\hline
\end{tabular}

with the middleleg amputated. When however, all values $T_{0}$ (except of that of OL 1 ) are higher or equal to 10.4 , any arbitrary order of values can be used. In this model there is no theoretical upper limit for these threshold values. The ranges of the other parameters are also shown in Table 1.

The different starting positions investigated are shown in Table 2. Although the starting behaviour of 
the model differs when the values of the parameters are changed within the ranges given in Table 1, this is not regarded in detail as no systematic experiments have been performed concerning the starting behaviour of the animals and therefore no direct comparison is possible.

As Graham (1972) recognized, 1st instar stick insects most often use a completely different gait, called gait I or a tripod gait. Here the protraction time is not constant but proportional to the duration of the period. Additionally the phase relations between different pairs of legs do not vary with speed as is the case in gait II. The proposed model can easily be changed to describe gait I in the following way : a) the positions of the switch points are no longer speed dependent but fixed. b) The rate of return of the relaxation oscillators ("protraction") is no more constant but inversely proportional to the walking speed. The results of the model changed in this way are shown in Fig. 4 by triangles. As with the experimental values they lie on a straight line with the slope 1 .

\section{Discussion}

The model is built up on assumptions that are in part supported by experimental results. The model describes the temporal coordination of a straight walking stick insect for both gaits. Additionally the model gives information about the local position of the tarsi relative to the body.

Curve walking is not examined in this paper. Stick insects seem to have two possible mechanisms to perform a curved walk. Graham (1972) observed occasionally an uncoupling between right and left legs. This results in different periods between the legs of both sides. In the model this could be done by an interruption of the three connections between right and left oscillators (Fig. 2). The different periods could be caused by different central influences on the retraction speed of right and left sides while maintaining a constant stride length. Graham (1972) in 1st instars and Jander (1978) in adults describe another type of curve walking where the temporal coordination of the leg remains the same, but the stride lengths of the legs on opposite sides are changed. This can be performed in this model by altering the retraction speed and the thresholds of left or right legs.

Another area, in which the model can be enlarged, which is not considered in this paper is given by the fact mentioned by Graham (1972) and Wilson (1966) that sometimes there can be seen a type of coordination, which is the mirror image of the normal type in gait II. This means that the pair L1-R2 and the pair L 2-R 3 are no longer protracting simultaneously, but rather the pairs R1-L2 and R2-L3. Such a coordi- nation can be produced by a mirror image connection between right and left oscillators. As both types of coordination occur in the same animal, one could assume that also both types of cross connections exist. In the model proposed here those influences would appear to be much weaker and both an increase in their strength and a change in across the body frequency are required to produce the two alternative asymmetries.

The model is not able to describe changes of the AEP, which are shown by Bässler (1972) in amputees, in animals walking under load (Bässler, 1977) or in animals walking up a vertical path (Cruse, 1976). Experiments of my own (Cruse, 1979) seem to indicate that the exact position of the AEP of a hindleg is determined by the position of the PEP of the leg infront of it. Therefore, the change of the AEP might be done by a mechanism, which is completely different from those used in the present model. This possibility and the step to step variations in AEP are also reasons for the fact that in the model the position of each tarsus at every instant is not given in absolute values relative to the animals body but is given relative to the AEP and the PEP of this leg. If these values are however considered as mean values over a large number of steps then it may be possible to regard them as being measured in a coordinate system that is fixed relative to the insects body as was done by Bässler (1972) and Cruse (1976).

This last consideration is based on the following interpretation of the model. The input value to the relaxation oscillator corresponds to the spike frequency applied on the retractor and protractor muscle systems or on the forces developped by these muscle systems. The output of the relaxation oscillator corresponds to the geometrical position of the leg. This is called a peripheral model (Graham, 1977a). Then the AEP and the PEP are defined geometrically by sense organs. The same is true for the switch positions shown in Fig. 3. It is however also possible to interpret the model in another way ("central model", see also Graham, 1977a). With this interpretation the whole relaxation oscillator is situated within the central nervous system. This means that the AEP and the PEP are not determined by sense organs but by internal values and that the switch positions (Fig. 3) are not determined geometrically but by central time delay mechanisms. The output value of the relaxation oscillator could be interpreted as the reference value of the servomechanism that moves the leg. As an alternative to this two extreme interpretations in the real biological system possibly a combination of both principles is realized e.g. that both principles work in parallel.

As in the above model and those already mentioned, as a simplifying assumption it is supposed that 
for a chosen walking speed the retraction speed of each single leg is constant during the whole retraction phase. This presumes that the total propelling force and the speed of the whole animal is constant at every moment. That this is not the case is shown for the speed by Graham (1972) and for the force by Cruse (1976). All these models therefore can only be regarded as first order approximations. As at the moment not enough exact data are available no better approximations have been tried in the model.

As mentioned in the introduction, there are two other quantitative models in the literature describing the coordination pattern of walking stick insects. What is the differenence between these models? The model of Graham $(1972,1977$ a) exists in two forms depending upon the precise definition of the oscillator. In the COM (central oscillator model) form it is a pure timing model suitable for an entirely endogenous system. In its peripheral form (POM or peripheral oscillator) the model uses a similar oscillator to that described here but the interactions are produced by increasing the thresholds. In both forms it provides the times of the onset of the protraction phases for a broad series of experiments at different speeds, for various amputations and is applicable to several insect species (stick insect, cockroach, locust, grasshopper). In its most abstract form the basic assumptions made are independent of any detailed morphological and physiological suppositions. However, it does permit speculation about such facts and can form the basis of more specialized models. So far as coupling between oscillators is concerned it is based firmly upon increased threshold interactions and an anteriorly directed information flow. These are the two principle differences between the model presented here and that of the Graham POM. I have been able to show that decreasing the threshold interactions between the oscillators and a rearward directed information flow can also in principle describe the basic locomotor patterns of the stick insect. Both models assume that the eigenfrequencies of the oscillators increase from the rear to the front. Another important distinction is that the above eigenfrequency relationship is only required for the highest eigenfrequencies. For certain low eigenfrequency exceptions see text relevant to Table 1 .

In Wendlers model $(1968,1978)$ as in the model of Graham the information flow is anteriorly directed. The nature of the connections between the six oscillators however is different. In Grahams model, as in the model proposed here, coupling is maintained by influencing the thresholds of the oscillators. In Wendlers model not the thresholds but the input to the relaxation oscillators is controlled and therefore the duration of the retraction phase is influenced. The coupling is done by an additive influence proportional to the output values of the controlling oscillators which correspond to the position of the different legs. In addition the number and arrangement of the connections between the six oscillators is very different to the model proposed here.

As mentioned earlier the model introduced here fails to describe all the properties of the walking insect. However, because of its rigorous construction experiments can be found that can test the assumed hypothesis. In all these experiments one must however be aware of the fact that the system under examination is a very adaptive one, even if the animal is intact and the walking situation is only slightly changed (Cruse, 1976). The stronger the experimental influence, and the amputation of a leg probably is a very strong influence, the more the system itself may be changed. When therefore a simple model such as those discussed here can no longer describe a new behaviour, this does not necessarily mean that some essential properties of the simple model may not still be found in the intact animal.

\section{References}

Bässler, U.: Zur Regelung der Stellung des Femur-Tibia-Gelenkes bei der Stabheuschrecke Carausius morosus in der Ruhe und im Lauf. Kybernetik 4, 18-26 (1967)

Bässler, U.: Zur Beeinflussung der Bewegungsweise eines Beines von Carausius morosus durch Amputation anderer Beine. Kybernetik 10, 110-114 (1972)

Bässler, U.: Sensory control of leg movement in the stick insect Carausius morosus. Biol. Cybernetics 25, 61-72 (1977)

Cruse, $\mathrm{H}$.: The function of the legs in the free walking stick insect, Carausius morosus. J. comp. Physiol. 112, 235-262 (1976)

Cruse, $H$. : The control of the anterior extreme position of the hindleg of a walking insect. Physiol. Entomol. (in press)

Cruse, H., Schwarz, G.: The coordination of oscillations of force in the standings legs of a walking insect (Carausius morosus). J. comp. Physiol. (submitted)

Graham, D.: A behavioural analysis of the temporal organisation of walking movement in the 1 st instar and adult stick insect (Carausius morosus). I. comp. Physiol. 81, 23-52 (1972)

Graham, D.: Simulation of a model for the coordination of leg movement in free walking insects. Biol. Cybernetics 26, 187-198 (1977a)

Graham, D.: The effect of amputation and leg restraint on the free walking coordination of the stick insect Carausius morosus. J. comp. Physiol. 116, 91-116 (1977b)

Graham, D.: Unusual step patterns in the free walking grasshopper Neoconocephalus robustus I. I. comp. Physiol. 73, 147-157 (1978a)

Graham, D.: Unusual step patterns in the free walking grasshopper Neoconocephalus robustus II. J. comp. Physiol. 73, 159-172 (1978b)

Graham, D.: Effects of circum-oesophageal lesion on the behaviour of the stick insect Carausius morosus. Biol. Cybernetics 32, $139-152(1979)$

Hughes, G.M.: The co-ordination of insect movement. Il. The effect of limb amputation and the cutting of commissures in the cockroach (Blatta orientalis). J. exp. Biol. 134, 306-333 (1957)

Jander, J.P.: Zur Kurventechnik laufender Insekten. Verh. Dtsch. Zool. Ges. 1978 (in press) 
Ten Cate, J.: Beiträge zur Innervation der Lokomotionsbewegung der Heuschrecke Locusta viridissima. Arch Néerl. Physiol. 21, 562-566 (1936)

Wendler, G.: Laufen und Stehen der Stabheuschrecke Carausius morosus. Z. vergl. Physiol. 48, 198-250 (1964)

Wendler, G.: Ein Analogmodell der Beinbewegungen eines laufenden Insekts. In: Kybernetik, 1968. Marko, H., Färber, G., eds. München, Wien: Oldenbourg 1968

Wendler, G.: Erzeugung and Kontrolle koordinierter Bewegungen bei Tieren. In : Kybernetik 1977. Hauske, G., Butenandt, E., eds. München, Wien : Oldenbourg 1978

Wilson, D.M.: Insect walking. Ann. Rev. Entomol. 11, 103-122 (1966)
Wong, R.K.S., Pearson, K.G.: Properties of the trochanteral hair plate and its function in the control of walking. J. exp. Biol. 64, 233-249 (1976)

Received: November 20, 1978

Prof. Dr. Holk Cruse

FB Biologie der Universität

Postfach 3049

D-6750 Kaiserslautern

Federal Republic of Germany 